\title{
Social Media Health Communication: A Cross-Cultural Investigation on the Motivations and Challenges of Using Participatory Technology to Communicate with Patients
}

\author{
L. Meghan Mahoney, West Chester University of Pennsylvania, USA \\ Bessie Lawton, West Chester University of Pennsylvania, USA \\ Lukas Pelliccio, West Chester University of Pennsylvania, USA
}

\begin{abstract}
The purpose of this study is to better understand how physicians utilize social media technologies to facilitate patient communication and the motivations and barriers towards doing so. Additionally, it explores how power distances influences physician's willingness to utilize social media as a resource in health communication by conducting in-depth interviews with physicians in the United States and in the Phillippines, based on divergent power distance scores from Hofstede's (2010) research. Qualitative analysis of transcripts revealed two major themes : 1) hesitancy in utilizing social media in doctorpatient communication for diagnosis and treatment; and 2) the role of social media for marketing, education, and communicating with hard-to-reach patients. These findings suggest that although physicians understand the many possible benefits of utilizing social media for health communication, there are many other factors to consider. Cultural issues should be taken into account as future social media health communication programs are developed.
\end{abstract}

Keywords: Social Media, Health Communication, Power Distance, Media Studies 


\section{Introduction}

Recent advances in new media technologies have greatly influenced the field of health communication. Though the health industry has proven slow to adopt to new technologies (DesRoches, Campbell, Rao, et. al., 2008), internet users have quickly subscribed to using the medium as the first resource when seeking health information. Currently, $80 \%$ of internet users utilize the internet to find health information (Pew Internet \& American Life Project, 2012). It is important to understand this informationexchange further to ensure that the process is effective to personal healthcare management.

Research demonstrates the importance of effective communication between physicians and patients as an essential element to positive healthcare perceptions (Haean, Ray \& Allegrante, 2008). Increased health communication provides audiences with more choice, both in their own personal health care management (Collins, Clark, Petersen \& Kressin, 2002), as well as in healthcare provider decisions and treatment advice (Haean, Ray \& Allegrante, 2008). While social media helps facilitate more efficient communication between physicians and patients, physicians have proven slow to adopt to the technology (see: Ford, Menachemi, \& Phillips, 2006; Lee, Cain, Young, et. al., 2005). The purpose of this study is to better understand how physicians utilize social media technologies to facilitate patient communication and the motivations and barriers towards doing so. Additionally, it will explore how power distances influences physician's willingness to utilize social media as a resource in health communication.

\section{Literature Review}

\section{Transformation of Health Communication Technologies}

New media technologies have shifted the linear broadcast model of communication to a more participatory and interactive process. While the internet began as a one-to-many model of communication technology (Web 1.0), early advancements led to more userbased interactivity with media content (Web 2.0). Web 2.0 content allows for users to find and keep in contact with personal community through social networking features 
(Boulos \& Wheeler, 2007). It allows for better interaction orientation where users are able to provide feedback and engage content; there is more personalization and customization of content based on user interest; and Web 2.0 allows for more user-added possibilities that transform users into producers of internet content (Wirtz, Schilke \& Ullrich, 2011). Overall, these Web 2.0 advancements in health communication have been found to be mostly positive additions to the health communication process.

These more interactive and participatory healthcare users often turn to social media in their pursuit of better healthcare information. Social media is defined as a group of internet-based applications that build on the ideological and technological foundations of Web 2.0 that allow the creation and exchange of user generated content (Kaplan \& Haenlein, 2010). Social media features include networking sites, blogs, collaborative projects, content communities and virtual worlds. Users are able to utilize social media to share their experiences with online communities, rate physician services and find alternative treatment recommendations. Physician attitudes towards utilizing social media for health communication have proven very split (Miller \& Sim, 2004) due to the many challenges and benefits the medium provides.

\section{Health Challenges and Benefits of Social Media}

Overall, physicians have cited patient privacy, standard of care, increased litigation and higher costs as barriers to utilizing social media for communication with patients (Haean, Ray \& Allegrante, 2008). Healthcare has traditionally been seen as a private-sphere topic. However, social media allows individuals a public forum to meet and connect with others who are struggling with the same health concerns. While this is great for sharing advice and experiences, it does create confidentiality concerns for physicians looking to engage in social media dialogue. Global laws about patient privacy varies, but the World Health Organization recognizes that it is an inherent right for all patients around the world the right to confidentiality of their personal health information (WHO, 2013). It is much easier to secure these conversations when they are held face-to-face in the privacy of a healthcare practice than it is to secure an online conversation. 
One critical health concern is the quality of health information consumed and diffused on the web, especially as the process becomes more social in nature. The internet is a largely unregulated space, where anyone is able to post anything on a designated topic. There are many sites with public health misinformation, some promoting products, and others as outright scams (Morahan-Martin \& Anderson, 2000). Each day, the amount of misinformation is ever growing. Some physicians worry about deadly consequences of spreading blatant misinformation online (Intlekofer, Cunningham \& Caplan, 2012, 39).

Decreased privacy and increased misinformation is a recipe for physician litigation. High numbers of malpractice lawsuits have resulted in many healthcare costs, both financially and in practice. Research demonstrates that litigation is responsible for over $\$ 30$ billion dollars in American healthcare costs (Searcey \& Goldstein, 2009). Often this fear of getting sued results in physicians taking fewer risks and getting less personal with patients during care.

Another concern as the healthcare industry moves towards the digital age is the increase in technology costs. Face-to-face communication and paper records are less expensive than new communication technologies. $81 \%$ of economists believe that the increased cost of healthcare is due to the process by which medical technologies are developed, adopted, and integrated into the delivery of health care services (Chernew, Hirth, Sonnad, et. al., 1998). These factors combined make many physicians uneasy about incorporating social media into their health communication plan, despite its many benefits.

To date, most health communication research has been focused on the benefits of patients accessing health-centered Web 1.0 websites. These sites have traditionally been the number one resource for users interested in being more proactive with their personal healthcare management (McMillan \& Macias, 2008). They are still designed as a one way model where users access information that is diffused in a top-down manner. 
However, social media allows users to share these more Web 1.0 oriented websites with their social networked Web 2.0 community.

There are many benefits of a more social online health experience. This freedom of information access increases audience knowledge, awareness and empowerment regarding personal health issue, equalizing information access. Social media is instant, borderless and elevates electronic communication to near face-to-face, changing diagnoses into dialogue (McNab, 2009). Social media is also found to result in greater patient happiness and a more patient-centered health care system (Hawn, 2009).

Research demonstrates how patients who are more proactive in personal health management are more willing to follow treatment advice (Berry, Parish, Janakiraman et al., 2008). Social media prompts more action from users. While it may be easy to dismiss what a physician has prescribed, it is less likely that one will ignore information sought through his or her own efforts.

By shifting the locus of control to the patient, relations are altered into more knowledgeable and empowered patients. Social media allows for user communities to share experiences about niche healthcare decisions (Sechrest, 2010). Blogs and online journals help provide patients with self-expressive tools to publish their own experiences and prompt treatment dialogue among niche communities (Hillan, 2003). Social media allows consumers to become creators and co-creators in the social marketing process by allowing for more public reporting, a key strategy in the effort to improve health care quality (Thackeray, Neiger, Hanson, et al., 2008). These types of social media provide another method for patients to provide feedback and obtain information about physician performance before making healthcare decisions (Lagu, Hannon, Rothberg, et. al., 2010).

Individuals are no longer limited to the expertise of the one or two physicians in their hometown. It is clear that the Internet is advancing more equal access to health communication across all populations. Patients who may otherwise hold limited access to 
healthcare providers are now using the technology for personal healthcare regiment advice. The same is true for other constraints, such as time, money or insurance. Online health dialogue has provided an opportunity for these individuals to seek health advice without physically making a trip to the doctor. In fact, the number of people seeking health information online is currently higher than the number of people seeking medical care in person (Bastian, 2008). It is imperative that physicians around the world are accessible and an intricate part of these conversations. However, many cultural differences may influence physicians' willingness to do so.

\section{Cultural Differences in Power Distance}

Culture is an imperative element of the human experience. It influences what is seen, what is remembered, and how information is processed (Oyserman, 2006). According to Hofstede's (1980) dimensions of culture, power distance plays a key role in the communicative process. It is defined as the extent to which the less powerful members of organizations and institutions accept and expect that power is distributed unequally. These normative cultural inequalities become imbedded into the fabric of everyday life when family structures, forms of government, architecture, religion, and other institutions are created under the collective mental programming of these cultural ideologies (Hofstede, 1980).

We have learned how new technologies hold great potential in dramatically influencing the relationship between patients and physicians (see: Kreuter, 2000; Neuhauser \& Kreps, 2003; and Weisbrod, 1991). Online users have evolved into more empowered, participating and active members of the healthcare process (see: Couchman, Forjuoh \& Rascoe, 2001; Roter \& Hall, 2006). Patients are using new media to research individual symptoms, discuss treatment options with online communities, and gain immediate access to second opinions and/or alternative physicians. However, not all cultures may view these changes in patients as positive advancements. Research suggests that collectivist cultures, like many in South East Asia, tend to adhere to norms of high power distance. Individualistic cultures, such as the United States and many European nations, 
tend to be low power distance societies (Hofstede, 2001; Merkin, 2006; Dysart-Gale, 2006). For collectivist cultures, individuals in positions of power are given the utmost respect, allowing for hierarchical displacement to become tangible in reality, so that differences in status are inherent and not human constructs.

One manifestation of power distance therefore is in the medical field, in the sense that cultures may accept or reject the notion of doctor superiority over patients differently, depending on whether the culture is generally accepting of social status hierarchies or not. For example, in a traditional doctor-patient relationship, the doctor generally has greater access to medical information, is presumed to hold expert medical knowledge, and has the role of dispensing medical information, diagnosing, and prescribing treatment to a generally dependent or passive patient. This relationship is generally unequal in status and power, but the rise of social media has led to the phenomenon of patients surfing for medical information and self-diagnosing, challenging the traditional model of medical care. This may have implications on how doctors relate to patients. Having awareness of the influence of power distance on a certain culture becomes a crucial concept in understanding communication between doctors and patients (Dysart-Gale, 2006).

Based on these understandings, this study was conducted to explore similarities and differences in United States and Philippine physician attitudes toward utilizing social media with their patients. The following research question has been advanced:

RQ: What motivations and barriers influence US and Filipino doctors' decisions to incorporate or not incorporate social media technologies into their practice?

\section{Methodology}

\section{Sample}

This study focuses on two countries with divergent power distance scores based on Hofstede's research. Hofstede, Hofstede, and Minkov (2010) report that the US scores 92 and the Philippines scores 162 on the power distance dimension. The Philippines' 
large positive score shows that the culture is high, while the US's low negative score reflects that it is low, on that dimension. Another reason why these countries were chosen for comparison is the fact that both countries have high levels of English literacy. In both countries, the study focuses on doctors in urban areas, where there are higher levels of social media penetration.

\section{Data analysis}

Qualitative interviews were analyzed following social science guidelines for qualitative data analysis. Interviewers took detailed notes while interviewing. Data was analyzed following Seidman's (2006) guidelines. Specifically, the process of identifying key themes from the interviews was done by reducing the text of interviews to what was most interesting for the research. "Most important is that reducing the data be done inductively rather than deductively ... the researcher must come to the transcripts with an open attitude, seeking what emerges as important and of interest from the text" (Seidman, 2006, p. 117).

Transcribed interview data was then repeatedly read and analyzed by individual researchers in order to identify relevant narratives that existed. Overall, this inductive process of coding meanings from interview transcripts resulted in two emergent themes that were useful in shedding light on the research question of the study.

\section{Results and Discussion}

Qualitative analysis of transcripts revealed two major themes relevant to the research question, namely: 1) hesitancy in utilizing social media in doctor-patient communication for diagnosis and treatment; and 2) the role of social media for marketing, education, and communicating with hard-to-reach patients (mainly nontypical patients such as those who lived in other countries). Hesitancy in using social media in doctor-patient communication. The study's research question asked how US versus Filipino doctors felt about using social media in their doctor-patient communication processes. Both US and Filipino doctors generally expressed hesitancy in using social media in diagnosis and 
treatment, but there were differences in the concerns they raised. Some barriers among Filipino doctors involved a strong desire for interpersonal contact with patients, work-life balance issues, preference for control of treatment, and frustration regarding the use of social media sites to rate doctors. In addition, legality/confidentiality was a major issue voiced mainly by US doctors.

One commonly voiced perspective among Filipino doctors but not by any US doctor was the importance of interpersonal touch and face-to-face communication with their patients. This is consistent with Koo and Choi's (2005) description of "Oriental" medicine as representing holistic worldviews. They describe a major focus of this medical perspective as developing relationships with the patient, because it is important to pay attention to the whole person, treating the patient as a whole entity instead of looking at specific symptoms as separate functions. Im, Ko, Hwang, and Chee (2012) and Kaptchuk (1982) also make the case that there is a difference between symptom-specific versus holistic treatment. Philippine physicians expressed views that emphasized getting to know the patient personally as an important part of diagnosis and treatment.

I'm very traditional so I'm not comfortable with new technologies with regard to patients. I want to see, feel, and touch. I prefer one on one communication with the patient because assessing the patient as a whole is personal, should get to know patient personally.

It has to be old-fashioned, consultation is something that should exist between a patient and a physician, whether by teleconference or office, that is the proper way to conduct a consultation. When it comes to consultation over the Internet like email, I don't participate in that kind of medical practice. Follow ups, still, esp. dealing with surgery, they have to come in, they cannot show me a picture of their incision over the web. I cannot make an intelligent assessment of what is going on.

In contrast, here's how a US physician described a typical appointment. 
Physicians and dentists don't spend enough time actually talking to the patient one on one. Even if it's just conversation about the weather or whatever, they're in and out. Wait in the waiting room for an hour and a half and you go in and the doc spends 15 minutes with you and there's not a lot of time for questions. Not a whole lot of one on one contact.

Another major concern voiced by Filipino doctors related to work-life boundary issues. Filipino doctors, specifically, were more concerned about patients contacting them at all hours of the day. US doctors were concerned about keeping the boundary between professional and personal separate, but unlike Filipino doctors, did not seem to be worried that patients would not respect their off times. One Filipino physician explained,

Doctors are resistant to using social media because most patients here if they get hold of your number, they don't stop calling. They don't give respect to the holidays, weekend, nighttime, they have to intrude into your privacy.

...but patients now the problem with them is they want doctors to be more connected to them and they want the doctor to be more accessible. So right now I think doctors are becoming more harassed. I know someone who limits her patients to 10 a day no matter how toxic.

Another concern shared by Filipino doctors was how the interactivity features of new media technologies allowed patients to post feedback and reviews about their doctors. One neurosurgeon did not mind the comments because they were predominantly positive and served as good marketing for his practice.

One time I tried to Google myself online, and I saw people communicating about their doctors and problems. I did see my name twice that they are exchanging what their doctor did. The patient has been writing praises about how we handled his brain tumor so that's been good publicity for us. 
However, many other Filipino doctors expressed dissatisfaction with their patients having the ability to utilize online communities to discuss and rate their physicians:

Sometimes they also post negative reactions to doctors' management... which are unfair for doctors like us because we can't respond to it.... Now doctors are forced to act like they are under the patient, not the authority.

As implied by this comment, part of the reason this was difficult for doctors is because it turns around the model of health provision where the doctor holds the power in the relationship. This vignette can be understood using the power distance concept of hierarchical privilege held by physicians over patients. Authors (2013) describe how Filipino doctors generally did not have any concerns about being perceived as "ineffective," testament to an attitudinal norm that reflects high power distance as it venerates doctors as experts who can solve the problem. In contrast, American doctors wanted patients to see them as "effective," reflecting a more equal level of interdependence between doctor and patient. Social media sites that are public have given patients a venue for rating doctors informally in the Philippines, something that was not welcome to these physicians.

The concern most US doctors were most vocal about related to legal and confidentiality issues as barriers to using social media for doctor-patient communication. In contrast, this was a non-issue for Philippine doctors. Almost every US participant explained that patient confidentiality is the primary concern of any outgoing communication. The Internet poses many challenges for practitioners because social media allows multidirectional communication allowing anyone to read and interact. One respondent said:

Most of the concern revolves around security and privacy. Privacy being the biggest concern because of physicians fearing that if they start having dialogue with patients or somehow there's a perception that they are having a discussion 
with a patient, that is a violation. If a patient wants to disclose something on a public forum or have lab data or health information online could someone somehow hack into that and get it. That's not isolated to social media but really the focus is on privacy.

In some cases, even when the physician makes an effort to hide someone's identity, the openness social media can result in indirect privacy violations. This raises a different set of communicative concerns.

(Privacy is) one of the biggest challenges... last year an ER doctor did not disclose the patient's name but used enough identifiers posted on her Facebook page and was flagged from the hospital and reprimanded by the state medical board because of the fact that people in the community knew who this patient was based on enough descriptors it got picked up on the news and the blogosphere Patients absolutely reach out to me on Facebook with questions. Usually I don't respond on Facebook. I usually respond and give a private email back because I don't think that medical information should be dispersed that way. I have a blog on my website as well and when people post questions there I generally email them back privately so that its not posted there.

One respondent explained that they had to get approval from their legal department before starting a personal blog. Another participant said, in reference to doctor/patient accessibility, "The biggest problem is that there's a lot of legal mumbo jumbo that says we shouldn't email patients."

Due to the constant fear of being sued, several of the participants explained the importance of having disclaimers on their digital outlets.

"I'm not required to have a disclaimer, [but] for legal purposes I have to be very clear that any kind of public information is not intended to diagnose, treat or 
promote any kind of illness. Anybody can sue anybody for anything. You have to be as careful as possible."

The fear of being sued proved to be a significant difference between American and Philippine interviews. Sison \& Palma-Angeles (1997) describe Filipinos as a nonconfrontational and non-litigious society. Lawyers in the Philippines have been shown to rarely represent public-interest healthcare cases (Mello, et. al., 2006). During interviews, American participants seemed to almost immediately respond to questions by incorporating some type of legal concern. Although Philippine participants addressed the importance of ethical standards and confidentiality, they were not as openly fearful about being sued.

\section{The Role of Social Media for Marketing and Education}

While doctors in both countries showed resistance to using social media for doctorpatient communication regarding diagnosis and treatment, there was a general perception that different types of social media might be useful for other purposes. Thus, some motivations doctors mentioned for using social media were marketing, communication with hard-to-reach (mainly international) patients, and control of medical information dispensed to patients.

Due to the concern for confidentiality and maintenance of professional relationship norms, a dominant theme among both US and Filipino doctors was that social media could be primarily used for advertising, acting as a sort of digital kiosk that dispenses conservative and generalized medical facts, and physician contact information. One Philippine respondent stated, "I think the power of the Internet is a good marketing tool for what I do. I use FB and social media to advertise." An optometrist whose business relies mainly on word of mouth explained,

I think the power of the Internet is a good marketing tool for what I do. I use FB and social media to advertise. With what I do, testimonials matter, so it's more 
with marketing Lasik, it's more the word of mouth, goodwill that you build on your patients. Really to begin the relationship. mostly as a marketing tool.

One US doctor especially felt that social media was a useful vehicle for letting not just patients, but other doctors, about new developments in their practice.

You can also use social media to market your social media or your practice. If you're the first one in the country doing a certain transplant or anything robotic kidney removal you may want to let the world know about that. It's not just for patients, it may even be for other doctors around the world saying we are now using this revolutionary technology to do this.

An American physician similarly responded, "The only reason I have Facebook is because there's so much traffic on Facebook...[and] to steer people to my website." Email consistently came up as an important tool for a practice to function efficiently. Whether it was reminders for appointments, follow-ups, or general information, email acted as an effective means to digitally communicate private information.

In terms of follow up, I would say that $95 \%$ of my follow up and ongoing follow up, is all done by email. That's been very good for me. Everybody aside from very elderly people who aren't at all computer savvy, everybody uses email. Email is a lot more private but the privacy concerns involving email are tremendously less than social media.

Of all social media, though, participants from both countries tended to agree that Skype was the most useful tool available. Facebook and Twitter are considered public forums where one must be extremely cognizant of outgoing communication. Email is very direct and mostly serves the goal of communicating written messages to facilitate logistical issues such as scheduling appointments. Among social media, physicians felt that Skype was the closest to actual human contact. It requires both parties to be online at the same 
time, accept a connection, and most importantly, provides video of the other party in real time. Respondents indicated that the use of Skype is not a result of obligation to trends in popular culture, the way that Facebook is, but rather, the medium is legitimately beneficial in certain cases, such as when a patient is overseas. One Filipino dermatologist explained,

I do Skype consultations and phone consultations. I've got patients oversees. I have patients in Europe Thailand and Australia. All over the United States. Skype is a great tool. It's almost the same thing as having a patient in your exam room. One area commonly motivation to use social media by Filipino doctors was its promise in providing medical information to patients. Several voiced wanting to have some control over what medical information their patients receive. One physician said, "I think the good thing about that is as a doctor you can post articles for information you want them to learn." Another explained,

Public health awareness, try to beef up some misinformation, a classic example is vaccination and autism. You've got celebrities out there saying that vaccines cause autism and a lot of confusion among patients and if you get a lot of doctors blogging and using social media the right message you can have doctors collaborating with groups to alert patients, like the swine flu, that was a big concern,

The desire for control of information among these doctors reflects a manifestation of power distance in the medical field. As mentioned earlier in the literature review, in a traditional doctor-patient relationship, the doctor generally has greater access to medical information, is presumed to hold expert medical knowledge, and has the role of dispensing medical information, diagnosing, and prescribing treatment to a generally dependent or passive patient. The relationship is greatly skewed in favor of power in the doctor's hands. Authors (2013) found that both Philippine and American doctors perceived today's patients to be more informed than those of years past; however, 
Philippine doctors viewed this as a challenge while U.S. doctors viewed it as an opportunity to initiate conversation. Filipino doctors perceived that the amount of misinformation found on the Internet created more problems than solutions. One Philippine doctor used the term "weaponry" to describe this information in the sense that it could battle with the doctor's knowledge base. It therefore made sense that they would prefer to have some measure of control over the medical knowledge their patients get.

\section{Conclusion}

This study provides an understanding of physician concerns for utilizing social media technologies to facilitate patient communication. It also examines the role power distance plays in their willingness and hesitancy towards doing so. Specifically, it explores the motivations and barriers that influence doctors' decisions to incorporate or not incorporate social media technologies into their practice. It compares attitudes between physicians in the United States and in the Philippines.

Results suggest that attitudes of healthcare professionals vary in regards to their willingness to incorporate social media to facilitate patient communication. Though most physicians understand the great advancements social media provides patients, specifically in regards towards creating more participatory and involved roles in the healthcare process (see: McNab, 2009; Hawn, 2009; Berry, Parish, Janakiraman et al., 2008), many physicians were hesitant to incorporate technology due to significant barriers in their own practice. These concerns are consistent with current health communication literature regarding mixed barriers and low new media adoption rates (DesRoches, Campbell, Rao, et. al., 2008). Specifically, results of this study found that utilizing social media for diagnosis and treatment purposes was the greatest concern for physicians from both regions.

Cultural key issues for Filipino physicians include a desire for interpersonal contact, work-life balance issues, and preference for control of treatment and frustration regarding the use of social media sites to rank doctors. US physicians were more concerned with a 
decrease in patient confidentiality, online misinformation, privacy issues and litigation concerns. These findings suggest that although physicians understand the many possible benefits of utilizing social media for health communication, there are many other factors to consider. Cultural issues should be taken into account as future social media health communication programs are developed.

Physicians from both regions proved much more willing to utilize social media for marketing, education, and communicating with hard-to-reach patients. The technological opportunities in these areas far outweighed more traditional methods. These areas did not present the same level of concern as utilizing technologies for diagnosis and treatment. However, they may not provide the same participatory benefits for patients wishing to take control of their personal healthcare management. More information regarding the benefits of utilizing social media for these areas is needed.

It is important to understand this information-exchange further to ensure that the process is effective to personal healthcare management. Further qualitative and quantitative research is needed to better understand the attitudes of health practitioners, as well as patients, in regards to their willingness to utilize social media for health communication. Overall, this study demonstrates that technology access, competence and infrastructure are not the only barriers physicians must overcome in order to implement social media to facilitate health communication with patients. This helps to further explain the many possibilities for physician low rates of new media adoption. Social media platforms bring many new concerns that prove much more complex than current positive health communication literature suggests. 


\section{References}

Bastian, H. (2008). Health literacy and patient information: Developing the methodology for a national evidence-based health website. Patient, Education and Counseling, 73(3), 551-561.

Berry, L., Parish, J., Janakiraman, R., Ogburn-Russell, L. et al. (2008). Patients' Commitment to Their Primary Physician and Why It Matters. Ann Fam Med, 6, 613.

Boulos, K., \& Wheeler, S. (2007). The emerging Web 2.0 social software: an enabling suite of sociable technologies in health and health care education. Health Information \& Libraries Journal, 24(1), 2-23.

Chernew, M., Hirth, R., Sonnad, S., Ermann, R., \& Fendrick, A. (1998). Managed Care, Medical Technology, and Health Care Cost Growth: A Review of the Evidence. Medical Care Research and Review, 55(3), 259-288.

Collins, T., Clark, J., Petersen, L., \& Kressin, N. (2002). Racial Differences in how patients perceive physician communication regarding cardiac testing. Med Care, 40(1), 27-34.

Couchman, G., Forjuoh, S., \& Rascoe, T. (2001). E-mail Communications in Family Practice: What Do Patients Expect? Journal of Family Practice, 50, 414-418.

DesRoches, C., Campbell, E, Rao, S, Donelan, K., Ferris, T., Jha, A. \& Blumenthal, D. (2008). Electronic Health Records in Ambulatory Care-A National Survey of Physicians. New England Journal of Medicine, 359(1), 50-60.

Ford, E., Menachemi, N., \& Phillips, M. (2006). Predicting the adoption of Electronic health records by physicians: When will health care be paperless?. Journal of the American Medical Informatics Association, 13(1), 106-112.

Haean, O., Ray, M., \& Allegrante, J. (2008). Perceptions of Health Care Provider Communication Activity Among American Cancer Survivors and Adults Without Cancer Histories: An Analysis of the 2003 Health Information Trends Survey (HINTS) Data. Journal of Health Communication, 13(7), 637-653.

Hawn, C. (2009). Take Two Aspirin and Tweet Me in the Morning: How Twitter, Facebook, and Other Social Media are Reshaping Health Care. Health 
Affairs, 28(2), 361-368.

Hillan, J. (2003). Physician Use of Patient-Centered Weblogs and Online Journals. Clinical Medicine \& Research, 1(4), 333-335.

Hofstede, G., Hofstede, G.J., \& Minkov, M. (2010). Cultures and organizations: Software of the mind: Intercultural cooperation and its importance for survival (3rded.). New York: McGraw-Hill.

Im, E.O., Ko, Y., Hwang, H., \& Chee, W. (2012). Symptom-specific or holistic: Menopausal symptom management. Health Care for Women International, 33(6), $575-592$.

Intlekofer, K, Cunningham, M. \& Caplan, A. (2012). The HPV vaccine controversy, Medicine \& Society, 14(1), 39-49.

Kaplan, A. M., \& Haenlein, M. (2010). Users of the world, unite! The challenges And opportunities of Social Media. Business Horizons, 53(1), 59-68.

Kaptchuk, T. (1982). The holistic logic of Chinese medicine. Science Digest, 92(11), 32-34, 107.

Koo, M., \& Choi, I. (2005). Becoming a holistic thinker: Training effect of Oriental medicine on reasoning. Personality and Social Psychology Bulletin, 31, 124-1272.

Kreuter, M. (Ed.). (2000). Tailoring Health Messages: Customizing Communication with Computer Technology: Routledge.

Lagu, T., Hannon, N., Rothberg, M., \& Lindenauer, P. (2010). Patients' Evaluations of Health Care Providers in the Era of Social Networking: An Analysis of Physician-Rating Websites. Journal of General Internal Medicine, 25(9), 942-946.

Lee, J., Cain, C., Young, S., Chockley, N., \& Burstin, H. (2005). The adoption gap: Health information technology in small physician practices. Health Affairs, 24(5), 1364-1366.

Authors (2013). The utility of new media technologies in doctor-patient communication and power distance. Paper presented at the National Communication Association conference in Washington, D.C. 
McMillan, S., and Macias, W. (2008), Strengthening the safety net for online seniors: Factors influencing differences in health information seeking among older Internet users, Journal of Health Communication, 13(8), 778.

McNab, C. (2009). What Social Media Offers to Health Professionals and Citizens. Bulletin of the World Health Organization, 87(8), 566-566.

Mello, M., Powlowski, M., Nañagas, J., \& Bossert, T. (2006). The role of law in public health: The case of family planning in the Philippines. Social Science \& Medicine, 63(2), 384-396.

Miller, R., \& Sim, I. (2004). Physicians' use of electronic medical records: barriers and solutions. Health affairs, 23(2), 116-126.

Morahan-Martin, J., \& Anderson, C. D. (2000). Information and misinformation online: Recommendations for facilitating accurate mental health information retrieval and evaluation. CyberPsychology \& Behavior, 3(5), 731-746.

National Cancer Institute. (2001). Making health communication programs work. Bethesda, MD.

Neuhauser, L., \& Kreps, G. L. (2003). Rethinking Communication in the e-Health Era. Journal of Health Psychology, 8(1), 7-23.

Pew Internet \& American Life Project (2012), What Facebook and Twitter mean for news. Retrieved October 10, 2013, from http://stateofthemedia.org/2012/mobiledevicesand-news-consumption-some-good-signs-for-journalism/what-facebookandtwitter-mean-for-news/.

Roter, D. and Hall, J. (2006). Doctors Talking with Patients/Patients Talking with Doctors: Improving Communication in Medical Visits, 2nd ed. Praeger: Westport, CT.

Searcey, D. \& Goldstein, J. (2009). Tangible and Unseen Health-Care Costs. Retrieved February 12, 2013, from http://online.wsj.com/article/SB125193312967181349.html

Sechrest, R. (2010). The Internet and the Physician-Patient Relationship. Clinical Orthopaedics and Related Research, 468(10), 2566-2571. 
Seidman, I. (2006). Interviewing as qualitative research: A guide for researchers in education and the social sciences (3rd ed.). New York: Teachers College Press.

Sison, A. J. G., \& Palma-Angeles, A. (1997). Business ethics in the Philippines. Journal of Business Ethics, 16(14), 1519-1528.

Thackeray, R., Neiger, B., Hanson, C., \& McKenzie, J. (2008). Enhancing Promotional Strategies Within Social Marketing Programs: Use of Web 2.0 Social Media. Health Promotion Practice, 9(4), 338-343.

Weisbrod, B. (1991). The Health Care Quadrilemma: An Essay on Technological Change, Insurance, Quality of Care, and Cost Containment. Journal of Economic Literature, 29(2), 523-552.

WHO (2013). Patients' Rights. World Health Organization. Retrieved October 22, 2013, from http://www.who.int/genomics/public/patientrights/en/.

Wirtz, B., Schilke, O., \& Ullrich, S. (2011). Strategic Development of Business Models:Implications of the Web 2.0 for Creating Value on the Internet. Long Range Planning. 1-19. 